\title{
Photoleukocoria Detection in Children
}

\author{
Sofia Costa ${ }^{1 *}$, Ana Sofia Vaz ${ }^{1}$, Inês Melo ${ }^{1}$, Guilherme Castela ${ }^{2}$, Sónia Silva ${ }^{1}$ and Manuel Brito ${ }^{1}$ \\ ${ }^{1}$ Paediatric Oncology Department, Hospital Pediátrico, Centro Hospitalar e Universitário de Coimbra, \\ Coimbra, Portugal \\ ${ }^{2}$ Ophtalmology Department, Centro Hospitalar e Universitário de Coimbra, Coimbra, Portugal
}

\begin{abstract}
Introduction: Leukocoria, known as "white pupil", can be present in several eye diseases, including retinoblastoma, a rare malignant eye tumour. It can be detected in photographs and is called photoleukocoria. The red reflex test is the best tool to detect leukocoria, and the American Academy of Paediatrics (AAP) recommends that it should be performed in all children's routine medical appointments in the first five years of live.
\end{abstract}

Objectives: Evaluate medical ability (paediatricians and general practitioners) to identify photoleukocoria and assess the use of photographs and red reflex test in children's appointments at point 0 and six months later.

\begin{abstract}
Methods: The authors did a prospective study that took place in hospitals and primary care units in the centre of Portugal, between June 2017 and October 2018. Ten photographs of children under 5 years of age, including two with photoleukocoria due to retinoblastoma, were showed to medical doctors. They answered two questionnaires, with 6 months apart, about the usefulness of red reflex test and photos to identify leukocoria in clinical practice. Data were analysed using SPSS, version $22(\alpha=0.05)$.

Results: One hundred and ten doctors participated in the study (55\% paediatricians and $50 \%$ residents). Photoleukocoria was identified by $94 \%$ of the doctors, with no difference between paediatricians and general practitioners. Seventy-eight per cent used the red reflex test, but only $43 \%$ do it in all children's medical appointments under five years old ( $34 \%$ paediatricians and $9 \%$ general practitioners, with $p=0.002$ ).

Seventy-two doctors (65\%) answered the second questionnaire, 6 months later. There was a rise of $18 \%$ in the number of doctors that use the red reflex test and $60 \%$ have used photographs, at least one time, in their clinical practice to detect photoleukocoria.

Conclusions: The majority of the doctors are able to identify photoleukocoria. However, some of them don't use the red reflex test in children's appointments, and only a minority use it accordingly to the AAP recommendations. Awareness of children's health care providers is essential to allow early leukocoria detection and subsequent diagnosis of retinoblastoma and other eye diseases. Early diagnosis in Retinoblastoma allows higher rates of survival and vision preservation, with less treatment toxicity.
\end{abstract}

\section{Introduction}

In the western world, the incidence of low vision for congenital causes is about 1-6/10.000 live births [1].

Leukocoria describes the clinical finding of a white pupillary reflex. It comes from the greek "leucos" (white) and "korê" (pupil), meaning "white pupil" and occurs when the structures form the visual axis (cornea, lens, vitreous and retina) turn opaque. It could be a sign of several childhood eye diseases, including retinoblastoma $(\mathrm{Rb})$, which is the most serious, as it can be life threatening [2]. Although very rare (incidence: $1 / 15.000$ to $1 / 20.000$ live births), $\mathrm{Rb}$ is the most common malignant primary intraocular tumour in children and the second most common in all ages. The onset is usually within the first five years of life and leukocoria is the first sign in $60 \%$ of cases [3]. When it is diagnosed in early intraocular stages, the probability of cure is greater than $80-90 \%$ [4]. Other children's diseases that could present with leukocoria are cataract (the most common treatable cause of visual impairment in newborns), retinal detachment, retinopathy of prematurity, intraocular infection (endophthalmitis) and Coat's disease. Usually, these children appear healthy and don't have symptoms, which also contribute to diagnosis delay [2].

*Corresponding author: Sofia Costa, Paediatric Oncology Department, Hospital Pediátrico, Centro Hospitalar e Universitário de Coimbra, Coimbra, Portugal

Accepted: November 28, 2020

Published online: November 30, 2020

Citation: Costa S, Vaz AS, Melo I, et al. (2020) Photoleukocoria Detection in Children. Arch Fam Med Gen Pract 5(1):161-164 
In clinical practice, the red reflex test (RRT) is part of the visual screening in childhood. It consists in the transmission of light from an ophthalmoscope through all the normally transparent parts of the eye. It is properly performed in a darkened room, projecting the light of the ophthalmoscope (with the lens power set at " 0 "), onto both eyes of the child 1 meter away, and then onto each eye at the same time at a distance of 50 centimetres, allowing the detection of leukocoria and other visual axis abnormalities. It should be observed in all positions of gaze to confirm symmetry in colour and intensity in both eyes.

The American Academy of Paediatrics (AAP) recommends the use of the RRT in all children's routine appointments in the first five years of life [5]. In this period, Portuguese children's routine appointments should occur when they are 15 days old, 1, 2, 4, 6, 9, 12, 15 and 18 months, and then 2, 3, 4 and 5 years. However, visual screening, including the RRT, is recommended only at birth, 2 and 6 months and 2 and 5 years [6]. Any abnormal red reflex or leukocoria should immediately be referred to a paediatric ophthalmologist, because visual prognosis depends on an earlier detection and diagnosis, rapid referral, and prompt treatment.

Leukocoria can be found through simple flash photography, a phenomenon known as photoleukocoria, because any process that prevents the flashlight of a camera from reaching the retina will produce photoleukosis. Photos can help in the detection of an abnormal red reflex, showing a white eye instead of the typically red eye. It doesn't replace RRT. Instead, it can be an extra tool in leukocoria screening, in spite of rare false positive cases $[2,7]$. Photos are an easy, economic and accessible method to everyone and could help in the leukocoria screening. Photographs taken from different angles and in dim light (usually indoors), can detect early ocular abnormalities, even small and peripheral retinoblastoma tumours. However, it is important to know that it doesn't work with double flash option. Smartphone cameras also enable parents to detect red reflex abnormalities and, in most cases, parents are the first ones to detect photoleukocoria $[2,7]$. In a study of 1632 patients with retinoblastoma, in about $80 \%$ of the cases the suspicion was raised by a relative who observed photoleukocoria in a photograph that led to the diagnosis [8].

There are few studies published in the literature evaluating the use of RRT by children's health care providers and their ability to detect leukocoria/photoleukocoria [9-12]. As leukocoria is an important sign of different eye diseases, and simple to identify, it is important to know how child's doctors are familiarized with leukocoria and how they manage it, in order to organize more awareness campaigns. The aims of our study are:

- Primary aims:

1) Evaluate if paediatricians (PA) and general practitioners (GP) use the RRT in their clinical practice; 2) How they do it; 3) Analyse if they are able to identify photoleukocoria;

- Secondary aims:

4) Compare the use of the RRT and the ability to identify photoleukocoria between PA and GP and between consult- ants and residents; 5) How they act towards red reflex abnormalities; 6) Compare the use of the RRT and the use of photos, to identify photoleukocoria, before and after our first approach.

\section{Materials and Methods}

The authors did a prospective study to analyse the ability of a group of physicians (PA and GP, including consultants and residents) to identify photoleukocoria. It took place in hospitals and primary care units between June 2017 and October 2018. The inclusion criteria were: 1) Participants must be PA and GP; 2) Working in the centre of Portugal and 3) Without knowledge of the study. Photos of children with ages between 0-5 years were taken, using a camera at 1 meter. Some of them were patients with retinoblastoma and leukocoria, while others had normal red reflex in both eyes. All parents signed an informed consent. Ten photographs (two with photoleukocoria) were showed to the group of doctors, and it was asked if they had detected any pupillary reflex abnormalities. The ability to recognize photoleukocoria $(0,1$ or 2 photographs) was registered. Then, they had to fill in a questionnaire to collect personal and professional data (age, gender, years of experience, specialty, workplace) and some information about the use of the RRT. Six months after the first questionnaire, they filled in a second one with the same questions and with new ones about the use of photographs in the leukocoria screening (annex 1 ). The ability to recognize photoleukocoria, the use of the RRT and its conditions were compared between PA and GP and between consultants and residents.

This study was approved by the Hospital's Ethical Commission.

Data were analysed using SPSS, version $22(\alpha=0.05)$. We performed the chi square test to observe differences between groups (consultants vs. residents and PA vs. GP). The Wilcoxon test was done to verify if there were differences between the use of the RRT and photographs, in clinical practice, at point 0 and six months later.

\section{Results}

One hundred and ten doctors participated in the study. Sixty (55\%) were PA and 50 (45\%) were GP. Half of them were residents and the median age was 31 years old. One hundred and three doctors (94\%) were able to identify photoleukocoria in two photographs (58 PA and 45 GP). There was no statistical difference in the ability to recognize photoleukocoria between PA and GP ( $96 \%$ vs. $90 \%, p=0.081)$ nor between residents and consultants ( $90 \%$ vs. $96 \% ; p=0.772$ ) (Table 1 ). Three doctors were able to identify photoleukocoria in one photograph and four couldn't recognize it in any of them. First questionnaire analysis showed (Table 1):

- 86 doctors (78\%) use the RRT in their clinical practice, however only $43 \%$ do it in all children's routine medical appointments under 5 years of age, with statistical difference between paediatricians (34\%) and primary care doctors (9\%), $\mathrm{p}=0.002$;

- 33 doctors (30\%) use the RRT only in the first year of life 
Table 1: Identification of photoleukocoria (PL) and use of the red reflex test.

\begin{tabular}{|c|c|c|c|c|}
\hline & Identification of PL & & Red reflex test & \\
\hline Paediatricians & $58 / 60(96 \%)$ & \multirow{2}{*}{$p=0.081$} & $51 / 60(85 \%)$ & \multirow{2}{*}{$p=0.058$} \\
\hline Primary Care Physicians & $45 / 50(90 \%)$ & & $35 / 50(70 \%)$ & \\
\hline Residents & $53 / 55$ (96\%) & \multirow{2}{*}{$p=0.772$} & $41 / 55(75 \%)$ & \multirow{2}{*}{$p=0.745$} \\
\hline Consultants & $50 / 55$ (90\%) & & $45 / 55$ (82\%) & \\
\hline Total & $103 / 110(94 \%)$ & & $86 / 110(78 \%)$ & \\
\hline
\end{tabular}

Table 2: Comparison between answers of the first and second questionnaires.

\begin{tabular}{|c|c|c|c|c|}
\hline$N=72$ & $1^{\text {st }}$ questionnaire & $2^{\text {nd }}$ questionnaire & & \\
\hline Red reflex test in all appointments $<5 y$ & $35 \%$ & $53 \%$ & $+18 \%$ & $p=0.033$ \\
\hline Dark room & $54 \%$ & $69 \%$ & $+15 \%$ & $p=0.071$ \\
\hline $\begin{array}{l}\text { Evaluate both eyes and then one eye at each } \\
\text { time }\end{array}$ & $28 \%$ & $38 \%$ & $+10 \%$ & $p=0.108$ \\
\hline Use of photographs to detect PL & - & $\begin{array}{l}60 \% \\
\text { (32\% once time } \\
22 \% \text { more than once } \\
6 \% \text { in all appointments) }\end{array}$ & $+60 \%$ & - \\
\hline
\end{tabular}

and $8 \%$ do it according to the Portuguese recommendations (at birth, 2 and 6 months; 2 and 5 years);

- From those that use the RRT (86), more than a half (57\%) uses a dark room when performing the RRT, $61 \%$ evaluate only one eye at each time, $9 \%$ evaluates both eyes simultaneously and $30 \%$ do both tests sequentially.

Six months later, only 72 (65\%) of the participants in the study answered the second questionnaire. There was an increase in the number of doctors that perform the RRT in all children's appointments under five years of age, using the correct technique (dark room, evaluation of one eye at each time and then both eyes simultaneously). Besides that, $60 \%$ have used, at least one time, photographs to detect photoleukocoria: $32 \%$ once time, $22 \%$ more than once and $6 \%$ in all appointments (Table 2).

\section{Discussion}

RRT is a good way to identify leukocoria and other children's eye anomalies, being a useful, easy to perform and lowcost test for the early detection of serious low vision diseases (retinoblastoma, cataract, retinal detachment, retinopathy of prematurity, intraocular infection, Coat's disease) [1,5]. Because of low incidence, these pathologies aren't probably a concern for most children's health care providers. This technique is very dependent on technical aspects (type of ophthalmoscope, ambient light, degree of pupillary dilatation), human factors (experience of the performer, collaboration of the child and parents) and tumour aspects (peripheral location of the tumour and disease severity). This could explain its less sensitivity for peripheral Rb lesions, that is only $16 \%$ [13]. However, sensitivity of the RRT is higher for congenital eye diseases $[1,7,11]$.

Using photos to detect photoleukocoria could complement and contribute to increase RRT sensitivity. In cell phones' cameras, a red eye reduction feature is included, so red reflex is prevented. It has to be deactivated to be able to detect photoleukocoria. With the advent of smartphone technologies, there are applications designed to help detect red reflex abnormalities, including leukocoria [7].

In our study, the majority of the doctors recognized photoleukocoria as a warning sign and know that it should be urgently referred to an ophthalmologist. Most of them use the RRT, but only a few do it correctly, less than a half use it in all children's routine appointments under 5 years of age and some $(22 \%)$ don't do it at all, which is consistent with other studies $[9,10,12]$. Those facts could result in a delay in diagnosing children's eye pathologies with consequent poor visual outcome, and life threatening, if $\mathrm{Rb}$ is present.

In Portugal, Paediatric Ophthalmologic learning is not mandatory, during Paediatrics or General Practitioner residency. Nevertheless, national recommendations include the performance of RRT, but only in specific ages, missing other opportunities for visual screening under 5 years of age $(4,9$, 12,15 and 18 months and 3 and 4 years). This could explain the improper training in ocular screening and lack of awareness for the importance of the RRT. When comparing the first and second questionnaires, there was an improvement in the performance and frequency in the use of RRT, according to AAP recommendations. Besides that, $60 \%$ of doctors started to use photos in their practice, improving leukocoria screening. With the participation in this study, those doctors became more aware of the RRT importance and leukocoria screening. Consequently, we understand that awareness campaigns for health care providers about leukocoria and RRT have huge importance, increasing rates of children's eye diseases early diagnosis. It is also recommended that Paediatric Ophthalmology training should be included during PA and GP residency. Like AAP, it's mandatory that Portuguese recommendations include the RRT in all routine medical ap- 
pointments before 5 years of age. That information should figure in the Paediatric Report Card of each child.

\section{This study has some limitations}

The number of participants doesn't reflect the proportion of PA, GP residents or consultants working in the centre of Portugal; 38 doctors didn't answer the second questionnaire, reducing the population of our study. Also, this second limitation may affect our results, as maybe those doctors didn't improve their RRT and that's why they didn't answer.

\section{Conclusions}

GP and PA play an important role in the detection of leukocoria, a sign presenting in severe ocular diseases, such as retinoblastoma. As they are more likely to appear within the first five years of life, screening in all appointments during this period becomes very important. Awareness campaigns, including health care providers and parents, could be lifesaving and are essential to increase early diagnosis with better visual outcomes. Recently, a national campaign was conducted in Portugal, alerting for the importance of the RRT as well as for the alarm signs that must be urgently observed by a paediatric ophthalmologist. It is important to change recommendations on visual screening in Portugal, and to include some paediatric ophthalmological training in PA and GP residency.

\section{Declarations}

All methods were carried out in accordance with relevant guidelines and regulations.

\section{Contributions}

Sofia Costa was responsible for the study and wrote the manuscript. Ana Sofia Vaz and Inês de Melo helped collect data. Guilherme Castela, Sónia Silva and Manuel Brito also helped collect data and made some corrections in the manuscript.

All of them were responsible for the design of the project.

\section{Acknowledgments}

We would like to thank the medical doctors of hospitals and primary care units who participated in our survey.

\section{Funding}

No funding was accepted.

\section{Ethics Approval and Consent to Participate}

The Ethics Committee of the Centro Hospitalar e Universitário de Coimbra granted ethics approval for the trial (25 November 2016). Written informed consent was obtained from participants.

\section{Consent for Publication}

Not applicable.

\section{Competing Interests}

The authors declare that they have no competing interests.

\section{Data Availability}

All data generated or analyzed during this study are included in this published article (and its supplementary information files).

\section{References}

1. Cagini C, Tosi G, Stracci F, et al. (2017) Red reflex examination in neonates: Evaluation of 3 years of screening. Int Ophthalmol 37: 1199-1204.

2. Asensio-Sánchez VM, Díaz-Cabanas L, Martín-Prieto A (2018) Photoleukocoria with smartphone photographs. Int Med Case Rep J 11: 117-119.

3. Singla P, Ley-Smith R, Maxwell P (2015) Importance of the red reflex test in the diagnosis of eye cancer. International Journal of Ophthalmic Practice 5: 6.

4. Epelman S (2012) Preserving vision in retinoblastoma through early detection and intervention. Curr Oncol Rep 14: 213-219.

5. American Academy of Pediatrics (2008) Red reflex examination in neonates, infants, and children. Pediatrics 122: 1401-1404.

6. Boas Práticas em Oftalmologia - Elementos Clínicos de Avaliação e Referenciação (DGS, 2008).

7. Khedekar A, Devarajan B, Ramasamy K, et al. (2019) Smartphone-based application improves the detection of retinoblastoma. Eye 33: 896-901.

8. Abramson DH, Beaverson K, Sangani P, et al. (2003) Screening for retinoblastoma: Presenting signs as prognosticators of patient and ocular survival. Pediatrics 112: 1248-1255.

9. Marcou V, Vacherot B, El-Ayoubi M, et al. (2009) Abnormal ocular findings in the nursery and in the first few weeks of life: A mandatory, yet difficult and neglected screening. Arch Pediatr 16: 38-41.

10. Muen WJ, Hindocha M, Reddy MA (2010) The role of education in the promotion of red reflex assessments. J R Soc Med Sh Rep 1: 46.

11. Perilli R, Lanci M, Romanzo A, et al. (2015) Screening eye diseases in babies: An Italian experience on 5000 healthy, consecutive newborns. Ann Ist Super Sanità 51:387-389.

12. Carlos A Leal-Leal, Hector Dilliz-Nava, Martha Flores-Rojo, et al. (2011) First contact physicians and retinoblastoma in Mexico. Pediatr Blood Cancer 57: 1109-1112.

13. Canadian Retinoblastoma Society (2009) National retinoblastoma strategy Canadian guidelines for care: Stratégie thérapeutique du rétinoblastome guide clinique canadien. Can J Ophthalmol 44: S1-88.

DOI: $10.36959 / 577 / 498$ 